\title{
Asociaciones de foraminíferos aglutinados de la Formación San Cayetano en el Cinturón Plegado de San Jacinto (Norte de Colombia)
}

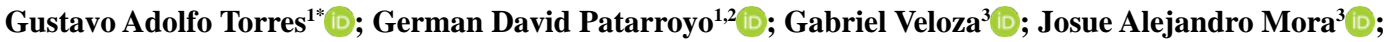 \\ Diego Fernando Gomez ${ }^{1}$
}

Forma de citar: Torres, G.A.; Patarroyo, G.D.; Veloza, G.; Mora, J.A.; Gomez, D.F. (2022). Asociaciones de foraminíferos aglutinados de la Formación San Cayetano en el Cinturón Plegado de San Jacinto (Norte de Colombia). Boletín de Geología, 44(1), 75-92. https://doi.org/10.18273/revbol.v44n1-2022003

\begin{abstract}
Resumen
El Paleógeno del Cinturón Plegado de San Jacinto (CPSJ) corresponde a una sucesión sedimentaria heterolítica, lo cual ha causado que el registro de microfósiles sea poco estudiado, particularmente para el Paleógeno Inferior. Este es el caso de la Formación San Cayetano, que por medio de las asociaciones de foraminíferos, ha sido interpretada como una unidad de edad Paleoceno-Eoceno temprano, y depositada en un ambiente de tipo turbidítico. Sin embargo, los niveles donde se han reportado los foraminíferos son intervalos discretos y un incremento en los estudios sedimentológicos y paleontológicos (macrofósiles), ha causado que tanto el rango cronoestratigráfico como los ambientes de depositación de la Formación San Cayetano vengan siendo objeto de debate. Teniendo en cuenta que las asociaciones de foraminíferos en esta unidad corresponden principalmente a formas bentónicas aglutinadas, se presenta el estudio taxonómico detallado de los especímenes más representativos en varias secciones que comprenden sedimentitas de la Formación San Cayetano. Las morfoespecies de foraminíferos descritas en este trabajo corresponden a especímenes poco diversos, con abundancias y preservaciones moderadas, los cuales comprenden principalmente formas aglutinantes tubulares y multiseriales. Aunque los taxones descritos representan en buena medida al tipo de foraminíferos reportados para la Formación San Cayetano, abarcando un intervalo de tiempo Paleoceno tardío-Eoceno temprano, algunos de los taxones referidos en este trabajo, como Aschemocella subnodosiformis, Bathysiphon eocanicus, Budhashevaella trinitatensis, Nothia lattissima o Reticulophragmoides jarvisi, ayudarían a restringir el rango de edad del nivel donde se encuentren. Igualmente, el tipo de foraminíferos encontrados confirma la interpretación previa de ambientes turbidíticos (flysch-type foraminiferal assemblages) o en su defecto, ambientes con una alta inestabilidad en el substrato. Por ende, estos resultados refuerzan la idea de que a futuro se clarifiquen las relaciones estratigráficas de los niveles de foraminíferos, con la de aquellos intervalos que han sido interpretados como depositados en ambientes proximales.
\end{abstract}

Palabras clave: Caribe colombiano; Foraminíferos bentónicos; Sistemática paleontológica; Paleógeno; Inferencias paleoambientales.

\section{Agglutinated foraminiferal assemblages of the San Cayetano Formation (San Jacinto Fold Belt, Northern Colombia)}

\begin{abstract}
The San Jacinto Fold Belt comprises a heterolithic succession, particularly in the Lower Paleogene, which might hamper the presence of diagnostic fossils. This case is notorious in the San Cayetano Formation, which strata have been interpreted as deposited in turbiditic setting and presents a late Paleocene-Early Eocene age using foraminiferal assemblages. However, the presence of foraminifers in the San Cayetano Formation is scattered, mostly constituted by benthic agglutinated assemblages, and the input of new data (sedimentology, macrofossils) have increased the discussion about the age and depositional context of that unit. Considering these issues, a detailed taxonomic study of the foraminiferal assemblages that define the Lower Paleogene in the San Jacinto Fold Belt was elaborated, using material of several sections of the San Cayetano Formation. Despite the agglutinated foraminifers present low diversity values, these have several abundance and preservation degrees, comprising tubular and multiserial agglutinated forms. Though some of the taxa have been referred in the San Cayetano formation, some of the identified species such as Aschemocella subnodosiformis, Bathysiphon eocanicus, Budhashevaella trinitatensis, Nothia lattissima or Reticulophragmoides jarvisi might refine the age proposal of late Paleocene-early Eocene. Furthermore, the foraminifers can be interpreted as flysch-type assemblages, or also associated with highly unstable substrates, aspects previously reported for this unit. In view of the most recent studies on the San Cayetano Formation, our contribution reinforces the importance of integrating paleontological and sedimentological data in incoming projects. This, in order of segregating the possible turbiditic settings of this unit from those proposed like more proximal.
\end{abstract}

Keywords: Colombian caribbean; Benthic foraminifers; Systematic paleontology; Paleogene; Paleoenvironmental inferences.

${ }^{1}$ Stratos Consultoría Geológica, Bucaramanga, Colombia. (*) gustshao@gmail.com; germanp@edu.unisinos.br; difergo86@yahoo.com

${ }^{2}$ Instituto Tecnológico de Paleoceanografía e Mudanças Climáticas, São Leopoldo, Brasil.

${ }^{3}$ Hocol S.A., Bogotá, Colombia. gabriel.veloza@hocol.com.co; alejandro.mora@hocol.com.co 


\section{Introducción}

Para la región del Caribe colombiano, y en particular en el Cinturón Plegado de San Jacinto (CPSJ), el registro de foraminíferos (bentónicos y plantónicos) ha sido fundamental para la definición del modelo de edad, las relaciones estratigráficas, y los ambientes de depositación de las distintas unidades litoestratigráficas que han sido propuestas en esa área (e.g. Redmond, 1953; Petters y Sarmiento, 1956; Chenevart, 1963; Stone, 1968; de Porta, 1970; Bordine, 1974; DuqueCaro, 1968, 1975; Guzmán et al., 2004; Rincón et al., 2007; Molinares et al., 2012; Osorio-Granada et al., 2020; Salazar-Ortiz et al., 2020a). Sin embargo, cuando se restringe ese acervo de información para la sucesión sedimentaria del Paleógeno, se observa que la aplicación de las asociaciones fósiles de foraminíferos tiende a ser restringida, en particular para la parte basal de la sucesión del Paleógeno (e.g. Formación San Cayetano; Duque-Caro, 1971a, 1971b; Guzmán et al., 2004; Molinares y Jaramillo, 2005; Patarroyo et al., 2019; Salazar-Ortiz et al., 2020b). La heterogeneidad composicional de las unidades litoestratigráficas presentes en el Paleógeno, al igual que una baja densidad de muestreo en comparación a las unidades que la subrayasen, pueden explicar el faltante de información micropaleontológica con base en foraminíferos.

En general, la bioestratigrafía para el Paleógeno del CPSJ, comienza con la definición de asociaciones de foraminíferos bentónicos por parte de Hermann Duque Caro en distintas publicaciones (Asociación Rzehakina epigona - Spiroplectammina grzybowskii; Duque-Caro, 1967, 1968, 1973; Duque-Caro et al., 1996). En trabajos más recientes, se ha buscado complementar dichas observaciones a través de estudios bioestratigráficos integrados (palinomorfos, nanofósiles, radiolarios, moluscos; e.g. GEOTEC, 2003; Guzmán et al., 2004; Rubio et al., 2009; Salazar-Ortiz et al., 2020b). Sin embargo, todos estos trabajos coinciden en los siguientes aspectos: 1) la dificultad de extraer elementos bioestratigráficos diagnósticos o con una amplia distribución geográfica en las sucesiones sedimentarias estudiadas, 2) una mayor recurrencia de los foraminíferos entre todos los microfósiles estudiados; en concreto, las asociaciones de foraminíferos bentónicos aglutinantes.

Si bien el uso de los foraminíferos bentónicos como marcadores bioestratigráficos genera debate por la dependencia facial que estos pueden presentar, diversas compilaciones ya muestran su aplicabilidad para el
Cenozoico de la región Caribe (e.g. Bolli et al., 1994). Por ende, la composición y distribución estratigráfica de las asociaciones de foraminíferos bentónicos referidas para la sucesión paleógena del CPSJ ameritan ser estudiados con mayor detalle. Este trabajo busca ampliar el conocimiento de las asociaciones reportadas para el Paleógeno en el CPSJ. Primero, a través de la descripción taxonómica detallada de algunos de sus taxones más representativos, generando un manual de referencia para futuros trabajos en el Caribe colombiano. Posteriormente, se actualiza el contexto bioestratigráfico de dichas asociaciones dentro de la Formación San Cayetano, así como las implicaciones paleoambientales de su presencia.

\section{El Paleógeno del Cinturón Plegado de San Jacinto}

Se ha documentado la convergencia oblicua y subducción de la placa del Caribe bajo la placa Sudamericana desde el Cretácico Superior hasta el Eoceno Inferior en el norte de Colombia (Mora et al., 2017a, 2017b). Dicha subducción generó un arco magmático en la actual Cordillera Central y en la cuenca del Valle Inferior del Magdalena. La Formación San Cayetano, definida en el Cinturón de San Jacinto al oeste del Valle Inferior del Magdalena, fue depositada entre el Paleoceno tardío y el Eoceno temprano, en una cuenca de antearco ubicada entre el arco magmático al este en el Valle Inferior, y la fosa de subducción ubicada al oeste. Esta unidad ha recibido varios otros nombres litoestratigráficos (e.g. Luruaco, Arroyo Seco) y ha sido interpretada por Mora et al. (2017b) como una secuencia estratigráfica de segundo orden (sensu Vail et al., 1977) que corresponde a un ciclo de alto rango/baja frecuencia (sensu Catuneanu et al., 2009). Con base en estudios de afloramiento (DuqueCaro, 1979, 1991; Guzmán et al., 2004; Salazar-Ortiz et al., 2020b), en recientes interpretaciones de sísmica 2D y en descripciones de núcleos de algunos pozos estratigráficos perforados por la Agencia Nacional de Hidrocarburos (ANH), se ha propuesto que la secuencia de la Formación San Cayetano se encuentra limitada por discordancias regionales angulares, una a la base sobre sedimentos cretácicos de la Formación Cansona y una al tope bajo sedimentos del Grupo Chengue del Eoceno Medio. También se ha propuesto que la Formación San Cayetano se depositó en ambientes que varían entre turbiditas arenosas en el sur, "fan deltas" en el centro y turbiditas principalmente lodosas en el norte del Cinturón de San Jacinto (Mora et al., 2017b). Las arenitas de la Formación San Cayetano 
se clasifican principalmente como litoarenitas, sublitoarenitas y arenitas feldespáticas con clastos de diversos tipos: ígneo plutónico y volcánico, metamórfico y sedimentario, incluyendo abundantes clastos de chert negro con foraminíferos planctónicos cuya proveniencia serían las capas de cherts Cretácicos de la Formación Cansona (Mora et al., 2017b). Análisis geocronológicos realizados a circones detríticos de la Formación San Cayetano en la zona norte revelan que los sedimentos de la unidad provienen de terrenos Permo-Triásicos y Cretácicos (Mora et al., 2017b), presentes en la Cordillera Central, en la Sierra Nevada de Santa Marta y documentados también en el basamento del Valle Inferior del Magdalena, al este del Cinturón de San Jacinto (Mora et al., 2017a).

\section{Área de estudio}

Las secciones (afloramientos y núcleos estratigráficos) y muestras puntuales de cartografía que fueron consideradas para el presente trabajo se encuentran distribuidas a lo largo de la parte media y sur del Cinturón Plegado de San Jacinto y comprenden sedimentitas que han sido identificadas como parte de la Formación San Cayetano (Figura 1).

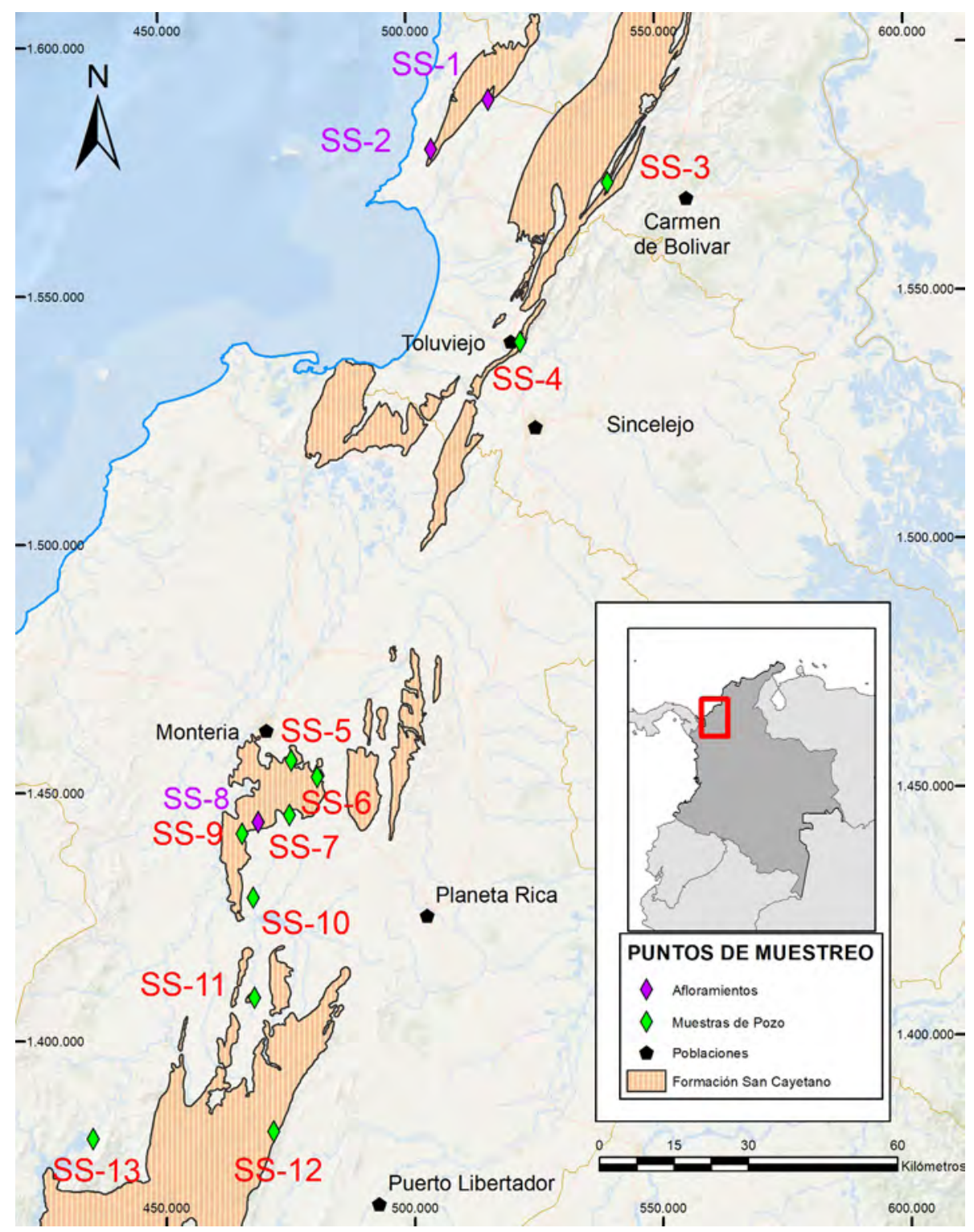

Figura 1. Localización del área de estudio y distribución de las secciones y núcleos estratigráficos respecto a los afloramientos de la Formación San Cayetano del noroccidente colombiano reportados por el Servicio Geológico Colombiano (Gómez et al., 2020). 


\section{Métodos}

Un total de 6 secciones (SS-3, SS-4, SS-5, SS-6, SS-12, SS-13) y 4 núcleos estratigráficos (SS-7, SS-9, SS-10, SS-11) que comprenden sedimentitas (principalmente limolitas arenosas y arcillolitas varicoroleadas) de la Formación San Cayetano fueron analizados en este estudio (Tabla 1). También se tuvieron en cuenta algunas muestras de superficie, las cuales serán referidas a lo largo del texto.

Tras disgregar las muestras con agua y peróxido de hidrógeno (5-10\%), los foraminíferos fueron asignados a una morfo-especie de ser posible, con el fin de identificar biozonas a lo largo de la sucesión sedimentaria.

Tabla 1. Localidades estudiadas del Paleógeno en el CPSJ.

\begin{tabular}{cccc}
\hline Localidad & Tipo de material & Coordenadas (X) & Coordenadas (Y) \\
\hline SS-1 & Cartografía & 846026 & 1585944 \\
SS-2 & Cartografía & 834540 & 1576003 \\
SS-3 & Afloramiento & 870010 & 1569306 \\
SS-4 & Afloramiento & 852480 & 1537276 \\
SS-5 & Afloramiento & 806459 & 1453198 \\
SS-6 & Afloramiento & 811673 & 1449869 \\
SS-7 & Núcleo estratigráfico & 806155 & 1442302 \\
SS-8 & Cartografía & 799819 & 1440848 \\
SS-9 & Núcleo estratigráfico & 796651 & 1438544 \\
SS-10 & Núcleo estratigráfico & 798852 & 1425708 \\
SS-11 & Núcleo estratigráfico & 799182 & 1405576 \\
SS-12 & Afloramiento & 803016 & 1378746 \\
SS-13 & Afloramiento & 766736 & 1377213 \\
\hline
\end{tabular}

Para la determinación de las morfoespecies reportadas y la revisión de las observaciones bioestratigráficas para cada grupo de muestras se tuvo en cuenta tanto literatura especializada a nivel global como de la región Caribe (e.g. Cushman y Renz, 1946; Loeblich y Tappan, 1987; Duque-Caro et al., 1996; Bolli et al., 1994; Kaminski et al., 1988; Kaminski y Gradstein, 2005; GEOTEC, 2003; Guzmán et al., 2004; SalazarOrtiz et al., 2020b). A su vez se consideraron las observaciones ecológicas de los géneros y las morfoespecies determinadas que permitieran ser relacionadas con un posible ambiente de depositación (Benedetti, 2017; Cetean et al., 2011; Murray et al., 2011; Setoyama et al., 2017).

\section{Resultados}

En general, los morfogrupos de foraminíferos corresponden a: 1) formas aglutinantes tubulares pertenecientes a los géneros Bathysiphon, Nothia, Psammosiphonella y Rhabdammina, 2) formas aglutinantes seriales pertenecientes a los géneros Ammobaculites, Archimerismus, Clavulinoides, Dorothia, Gaudryina, Karreriella, Marssonella,
Martinottiella, Pseudonodosinella, Pseudotextularia, Reophax, Textulariella, y Verneunilinoides, 3) formas aglutinantes espirales aplanadas pertenecientes a los géneros Ammodiscus, Glomospira, Phenacophragma y Trochamminoides, 4) formas aglutinantes planospirales pertenecientes a los géneros Budashevaella, Cyclammina, Evolutinella y Haplophragmoides, 5) formas aglutinantes trocospirales pertenecientes a los géneros Recurvoides y Trochammina, 6) formas aglutinantes globulares pertenecientes a los géneros Lagenammina, Psammosphaera y Saccammina y 7) formas aglutinantes elongadas quilladas pertenecientes a los géneros Spiroplectammina y Textularia.

Teniendo en cuenta que el recobro de foraminíferos fue muy variable en las muestras estudiadas (10200 ejemplares por muestra), y que el propósito fundamental de este trabajo es ofrecer una guía de trabajo para aquellos interesado en estudiar las formas aglutinadas de la Formación San Cayetano, el capítulo de sistemática paleontológica solo tendrá en cuenta las morfoespecies aglutinadas más representativas y aquellos especímenes de notable preservación de las secciones y núcleos estratigráficos estudiados. 


\section{Sistemática paleontológica}

Filo Foraminifera

Clase Globothalamea

Subclase Textulariana

Orden Lituolida

Suborden Trochamminina

Superfamilia Trochamminoidea

Familia Trochamminidae

Subfamilia Trochammininae

Género Ammoanita

Especie tipo

Ammoanita ruthvenmurrayi (Cushman y Renz, 1946)

Lámina 1, Figuras 1a-c

Material: 1 espécimen de la sección SS-3 (muestra SS3-419).

Descripción: Conchilla trocoespiral enrollada, hasta cuatro vueltas, biconvexa con periferia aguda. Contorno circular a ligeramente lobulado. Lado dorsal más fuertemente convexo que el ventral. El lado ventral presenta una depresión umbilical, suturas deprimidas y rectas. Cámaras numerosas, incrementado ligeramente en tamaño con 7-8 en la última vuelta. Ultimas cámaras más infladas que las primeras. Pared finamente aglutinada, a menudo bien silicificada. Apertura extraumbilical, redondeada, pequeña en la base de la última cámara.

Observaciones: El espécimen se corresponde con las características diagnósticas de Kaminski y Gradstein (2005). Sin embargo, difiere de las 7-8 cámaras originalmente descritas, presentando 6 cámaras en la última vuelta.

Edad: Campaniano a Paleoceno de acuerdo con Kaminski y Gradstein (2005).

Suborden Lituolina

Superfamilia Recurvoidoidea

Familia Ammosphaeroidinidae

Subfamilia Recurvoidinae

Género Budashevaella

Especie tipo

Budashevaella multicamerata (Voloshinova y Budasheva, 1961)

Lámina 1, Figuras 2a-b

Material: 1 espécimen de la sección SS-3 (muestra SS3-77+0,5).

Descripción: Conchilla grande, evoluta, con una periferia estrechamente redondeada y suturas marcadas. Enrollamiento inicial estreptoespiral y finalmente planospiral, con 2 a 2,5 vueltas apreciables y 10 a 18 cámaras en la última vuelta. Cámaras ligeramente infladas y suturas en la parte planospiral son rectas.
Cámaras en la parte inicial estreptoespiral enrolladas en ángulo alto respecto a la parte planospiral, claramente visibles en la región umbilical. Pared gruesa, media a finamente aglutinada a menudo silicificada. Cara apertural baja y apertura en hendidura interomarginal. Observaciones: El espécimen de $B$. multicamerata colectado presenta un tamaño similar al de ejemplares de B. trinitatensis de la misma sección. Sin embargo, las características diagnósticas en Kaminski y Gradstein (2005) pueden ser identificadas.

Edad: Eoceno medio a Mioceno de acuerdo con Kaminski y Gradstein (2005).

Especie tipo

Budashevaella trinitatensis (Cushman y Renz, 1946) Lámina 1, Figuras 3a-c

Material: 1 espécimen de la sección SS-3 (SS3-450).

Descripción: Conchilla pequeña a mediana, involuta o ligeramente evoluta, contorno ovalado con periferia redondeada. Enrollamiento inicial estreptoespiral y finalmente planospiral, con 6,5 a 8,5 cámaras en la última vuelta. Suturas rectas, no marcadas a ligeramente cortas y revelándose al humedecerse como bandas anchas y oscuras. Pared gruesa, finamente aglutinada y definida. Apertura en hendidura en la cara apertural, ligeramente sobre la base de la última cámara, rebordeada por un labio delgado.

Observaciones: La conchilla del espécimen colectado presenta deformación. No obstante, las características diagnósticas descritas en Kaminski y Gradstein (2005) pueden ser identificadas.

Edad: Paleoceno a Eoceno medio de acuerdo con Kaminski y Gradstein (2005).

Superfamilia Lituoloidea

Familia Haplophragmoididae

Género Haplophragmoides

Especie tipo

Haplophragmoides walteri (Grzybowski, 1898)

Lámina 1, Figuras 4a-c

Material: 1 espécimen en la muestra de cartografía SS-1.

Descripción: Conchilla planospiral de contorno circular, con periferia aguda. Su enrollamiento es involuto con 8 o 9 cámaras en la última vuelta. Cámaras triangulares que incrementan rápidamente en tamaño. Suturas rectas a sigmoidales y ligeramente deprimidas. Pared finamente aglutinada y definida

Observaciones: La conchilla del espécimen colectado presenta fragmentación en la periferia de las últimas cámaras. No obstante, las características diagnósticas descritas por Kaminski y Gradstein (2005) pueden ser identificadas. 
Edad: Cretácico tardío (Cenomaniano) a Eoceno tardío de acuerdo con Kaminski y Gradstein (2005). En Trinidad el rango de Haplophragmoides walteri (Grzybowski) corresponde al Paleoceno medio a Eoceno temprano (Bolli et al., 1994).

Superfamilia Lituotuboidea

Familia Trochamminoidae

Género Trochamminoides

Especie tipo

Trochamminoides proteus (Karrer, 1866) emend. Rögl, 1995

Lámina 1, Figuras 5a-b

Material: 1 espécimen de la sección SS-4 (muestra SS4-90).

Descripción: Conchilla casi planospiral en su última porción de contorno generalmente ovalado, lateralmente comprimida, compuesta de varias vueltas. El enrollamiento inicial es más irregular. En la última parte de la conchilla, las cámaras son globulares, redondeadas e incrementan moderadamente en tamaño. En los especímenes grandes, la penúltima vuelta tiene típicamente de 6 a 9 cámaras y en especímenes muy grandes 12 cámaras en la última vuelta. Pared finamente aglutinada de superficie lisa. Apertura interomarginal en arco amplio.

Observaciones: El espécimen en la muestra presenta deterioro de la conchilla. Sin embargo, las características diagnósticas de Kaminski y Gradstein (2005) pueden ser identificadas.

Edad: Campaniano a Eoceno de acuerdo con Kaminski y Gradstein (2005). En Trinidad el rango de Trochamminoides proteus (Karrer, 1866) corresponde al Maastrichtiano temprano a Paleoceno tardío (Bolli et al., 1994).

\section{Especie tipo}

Trochamminoides variolarius (Grzybowski, 1898)

Lámina 1, Figuras 6a-b

Material: 1 espécimen de la sección SS-3 (muestra SS3-397).

Descripción: Conchilla pequeña, compuesta de dos vueltas, con 4 cámaras en la última vuelta, cuadrada en contorno. Enrollamiento planospiral a trocoespiral bajo. Cámaras incrementando en tamaño rápidamente, algo alargadas, pero aumentando en diámetro hacia el centro. Pared finamente aglutinada, de superficie lisa, con la conchilla generalmente comprimida.

Edad: Cretácico tardío (Cenomaniano) a Eoceno de acuerdo con Kaminski y Gradstein (2005).

Suborden Spiroplectamminina
Superfamilia Spiroplectamminoidea

Familia Spiroplectamminidae

Subfamilia Spiroplectammininae

Género Spiroplectammina

Especie tipo

Spiroplectammina spectabilis (Grzybowski, 1898), emend. Kaminski, 1984

Lámina 1, Figuras 7-9

Material: 3 especímenes del núcleo estratigráfico SS10 (602,71 m), 2 especímenes de la sección SS-3 (SS327 y SS3-77+0,5) y 1 espécimen de la sección SS-6 (SS6-462,5).

Descripción: Conchilla inicialmente planospiral, luego biserial. En formas megaloesféricas, la porción planospiral consta de 4-7 cámaras, y puede ser más ancha que la porción biserial posterior. El segmento planospiral de las formas microesféricas es diminuta en comparación, y también puede ser más ancha que la parte biserial. Las cámaras en la parte biserial son bajas y numerosas. Suturas no marcadas a ligeramente cortas. En la parte biserial, suturas inclinadas alrededor de $60^{\circ}$ al eje longitudinal de la conchilla. El margen periférico es agudo y puede ser dentado o débilmente quillado. La pared es imperforada, finamente aglutinada de superficie lisa.

Observaciones: Algunos especímenes se encuentran fragmentados; No obstante, las características diagnósticas descritas en Kaminski y Gradstein (2005) pueden ser identificadas.

Edad: Maastrichtiano a Eoceno tardío de acuerdo con Kaminski y Gradstein (2005). En Trinidad el rango de Spiroplectammina spectabilis (Grzybowski, 1898) corresponde al Maastrichtiano temprano a Eoceno temprano (Bolli et al., 1994).

Orden Loftusiida

Suborden Loftusiina

Superfamilia Loftusioidea

Familia Cyclamminidae

Subfamilia Alveolophragmiinae

Género Reticulophragmium

Especie tipo

Reticulophragmium amplectens (Grzybowski, 1898)

Lámina 1, Figura 10a-c

Material: 1 espécimen de la sección SS-3 (muestra SS3-185).

Descripción: Conchilla biconvexa, planospiral involuta. Discoidal en vista periférica, con un ombligo poco deprimido. La conchilla es más gruesa alrededor del ombligo y se estrecha abruptamente hacia el margen periférico. Contorno redondeado 
a lobular en vista lateral. Aproximadamente dos vueltas y de 10 a 13 cámaras en la última vuelta en formas macrosféricas. Suturas rectas a ligeramente recurvadas y deprimidas en la última vuelta. Apertura en una hendidura interomarginal con labio fino. Pared finamente aglutinada bien cementada.

Observaciones: El espécimen corresponde a las características diagnósticas de Kaminski y Gradstein (2005).

Edad: Eoceno temprano tardío a Oligoceno temprano de acuerdo con Kaminski y Gradstein (2005).

\section{Género Reticulophragmoides}

Especie tipo

Reticulophragmoides jarvisi (Thalmann, 1932), emend. Gradstein y Kaminski, 1989

Lámina 1, Figura 11a-c

Material: 1 espécimen de la sección SS-3 (muestra SS3-15+0,5).

Descripción: Conchilla planospiral, biconvexa, con un engrosamiento umbilical, y 6 a 9 cámaras en la última vuelta. Periferia aguda, contorno circular a ligeramente lobulado, quillado. Suturas deprimidas, ligeramente curvadas y marcadamente limbadas. El lado umbilical tiene una protuberancia bien desarrollada. Apertura interomarginal sin labios ni aperturas suplementarias. Pared finamente aglutinada, con mucho cemento.

Edad: Paleoceno tardío de acuerdo con Bolli et al. (1994).

Orden Textulariida

Suborden Textulariina

Superfamilia Eggerelloidea

Familia Eggerellidae

Subfamilia Dorothiinae

Género Marssonella

Especie tipo

Marssonella oxycona var. trinitatensis Cushman y Renz, 1946

Lámina 1, Figuras 12a-b

Material: 1 espécimen de la muestra de cartografía SS-8.

Descripción: Esta variedad difiere de la típica en su forma pronunciadamente cónica, extremo inicial agudo y una apertura en arco alto.

Observaciones: Características similares a las descritas en Cushman y Renz (1946) y Bolli et al. (1994). Sin embargo, la disposición de las últimas cámaras sugiere una apertura en arco.

Edad: Campaniano a Paleoceno tardío de acuerdo con Bolli et al. (1994).
Clase Tubothalamea

Orden Spirillinida

Suborden Ammodiscina

Superfamilia Ammodiscoidea

Familia Ammodiscidae

Subfamilia Ammodiscinae

Género Ammodiscus

Especie tipo

Ammodiscus tenuissimus Grzybowski, 1898

Lámina 1, Figuras 13a-b

Material: 1 espécimen de la sección SS-3 (muestra SS3-419).

Descripción: Conchilla circular a ligeramente elíptica, delgada y puede tener un prolóculo globular. Enrollamiento planospiral ligeramente involuto, compuesto por hasta 7 vueltas. Cada vuelta va incrementando en diámetro lentamente al principio, luego más rápidamente. La última vuelta notablemente más ancha y superpuesta en las anteriores mientras que las suturas del enrollamiento están deprimidas. Pared delgada, finamente aglutinada con un acabado liso.

Observaciones: El espécimen presenta un enrollamiento involuto y suturas definidas, conchilla comprimida y pared finamente aglutinada que son características distintivas para la especie de acuerdo a Kaminski y Gradstein (2005).

Edad: Cretácico tardío (Cenomaniano) a Eoceno de acuerdo con Kaminski y Gradstein (2005).

Clase Nodosariata

Subclase Hormosinana

Suborden Hormosinina

Superfamilia Hormosinoidea

Familia Aschemocellidae

Género Aschemocella

Especie tipo

Aschemocella subnodosiformis (Grzybowski), emend. Kaminski y Geroch, 1993

Lámina 1, Figura 14

Material: 1 espécimen de la muestra de cartografía SS-8.

Descripción: Conchillas tubulares finamente aglutinadas con irregulares constricciones anulares, aunque el grado de constricción puede variar.

Observaciones: El espécimen en la muestra se encuentra fragmentado; No obstante, las características diagnósticas descritas en Kaminski et al. (1988) pueden ser identificadas.

Edad: Paleoceno temprano a Eoceno temprano de acuerdo con Bolli et al. (1994). 
Familia Hormosinidae

Subfamilia Hormosininae

Género Hormosina

Especie tipo

Hormosina cf. velascoensis (Cushman, 1926)

Lámina 1, Figura 15

Material: 1 espécimen de la sección SS-6 (muestra SS6-430+1).

Descripción: Conchilla robusta, uniserial, generalmente con hasta 5 cámaras, rara vez más. Cámaras cilíndricas y ligeramente fusiformes, a menudo están comprimidas lateralmente. Apertura terminal en amplia apertura circular. Pared finamente aglutinada, formada por múltiples capas de limo fino, generalmente silicificadas y criptocristalinas.

Observaciones: El espécimen reportado se encuentra fragmentado. Sin embargo, la conformación de las cámaras y la textura de la conchilla pueden ser identificadas de acuerdo con las características diagnósticas descritas en Kaminski et al. (1988).

Edad: Campaniano a Oligoceno de acuerdo con Kaminski y Gradstein (2005). En Trinidad el rango de Hormosina velascoensis (Cushman, 1926) abarca del Maastrichtiano tardío al Eoceno temprano (Bolli et al., 1994).

Clase Monothalamea

Orden Astrorhizida

Suborden Astrorhizina

Superfamilia Astrorhizoidea

Familia Rhabdamminidae

Subfamilia Bathysiphoninae

Género Bathysiphon

Especie tipo

Bathysiphon eocenicus Cushman y Hanna, 1927

Lámina 1, Figura 16

Material: 3 especímenes de la sección SS-6 (muestra SS6-430+1).

Descripción: Conchilla tubular, donde la pared tiene a menudo una apariencia calcárea y contiene granos de arena fina. Diámetro de 0,25-0,8 $\mathrm{mm}$.

Observaciones: El espécimen se corresponde con las características diagnósticas de Bolli et al. (1994).

Edad: Paleoceno temprano a Eoceno temprano según Bolli et al. (1994).

\section{Género Nothia}

Especie tipo

Nothia excelsa (Grzybowski, 1898), emend. Geroch y Kaminski, 1992

Lámina 1, Figuras 17-18

Material: 2 especímenes de la sección SS-6 (muestra SS6430+1) y 1 espécimen de la sección SS-13 (SS13-42).
Descripción: Conchilla de tamaño moderado, tubular, raramente bifurcada, sin prolóculo, o superficie de fijación, generalmente aplanada. Pared fina a moderadamente gruesa, bilamelar, con un acabado moderadamente rugoso y pudiendo contener algo de material pelítico. La mayoría de los fragmentos son rectos, pero otros pueden ser ligeramente curvos, retorcidos o ramificados.

Observaciones: Los especímenes en las muestras se encuentran fragmentados; sin embargo, las características diagnósticas descritas por Kaminski et al. (1988) pueden ser identificadas.

Edad: Cenomaniano a Paleogeno de acuerdo con Kaminski y Gradstein (2005).

Especie tipo

Nothia latissima (Grzybowski, 1898)

Lámina 1, Figura 19

Material: 1 espécimen de la muestra de cartografía SS-1.

Descripción: Conchilla tubular ancha y comprimida con una pared delgada, finamente aglutinada.

Observaciones: Los especímenes en las muestras se encuentran fragmentados; sin embargo, las características diagnósticas de Kaminski et al. (1988) pueden ser identificadas.

Edad: Campaniano a Paleoceno de acuerdo con Kaminski y Gradstein (2005).

Especie tipo

Nothia robusta (Grzybowski, 1898)

Lámina 1, Figuras 20-21

Material: 3 especímenes de la sección SS-6 (SS6$430+1)$ y 2 especímenes en el núcleo estratigráfico SS10 (239-9 m).

Descripción: Conchilla robusta, generalmente recta, aunque rara vez se encuentran fragmentos ramificados. La conchilla no presenta abultamientos o constricciones notables y podría aplanarse con un surco medio. Pared gruesa, media a finamente aglutinada, hecha de granos de cuarzo, con una superficie lisa, a menudo bien silicificada. La conchilla puede contener espículas de esponja orientadas al azar y ocasionalmente pueden presentar granos finos de minerales máficos en su superficie.

Observaciones: Los especímenes en las muestras se encuentran fragmentados; sin embargo, las características diagnósticas de Kaminski et al. (1988) pueden ser identificadas.

Edad: Cenomaniano a Mioceno temprano de acuerdo con Kaminski y Gradstein (2005). 
Género Psammosiphonella

Especie tipo

Psammosiphonella discreta (Brady, 1881)

Lámina 1, Figura 22

Material: 1 espécimen de la muestra de cartografía SS-2.

Descripción: Conchilla recta, no ramificadas, con constricciones anulares a intervalos irregulares, pero sin divisiones o cámaras internas centrales. En especímenes grandes puede presentar hasta 5 constricciones, pudiéndose estrechar hacia un extremo. La pared está compuesta de minerales (principalmente cuarzo), de granos firmemente cementados. El tamaño de grano del material aglutinado es variable. Apertura abierta al extremo del tubo.

Observaciones: El espécimen reportado se encuentra fragmentado. Sin embargo, características diagnósticas de Kaminski et al. (1988) como constricciones en la conchilla y cementación de granos gruesos en la pared pueden ser identificadas.

Edad: Cretácico a reciente de acuerdo con Kaminski y Gradstein (2005).

Suborden Saccamminina

Superfamilia Saccamminoidea

Familia Saccamminidae

Subfamilia Saccammininae

Género Saccammina

Especie tipo

Saccammina grzybowskii (Schubert, 1902)

Lámina 1, Figura 23

Material: 1 espécimen de la sección SS-6 (muestra SS6-478,4).

Descripción: Conchilla unilocular, de contorno casi circular, generalmente comprimida. Apertura proyectada en un cuello, usualmente localizada en la periferia de la conchilla. La rugosidad de la pared varía de mediana-gruesa a finamente aglutinada.

Observaciones: El espécimen se corresponde con las características diagnósticas descritas en Kaminski et al. (1988).

Edad: Cretácico temprano al Neógeno de acuerdo con Kaminski y Gradstein (2005). En Trinidad el rango de S. grzybowskii (Schubert, 1902) corresponde al Maastrichtiano tardío a Paleoceno (Bolli et al., 1994).

Superfamilia Psammosphaeroidea

Familia Psammosphaeridae
Subfamilia Psammosphaerinae

Género Psammosphaera

Especie tipo

Psammosphaera irregularis (Grzybowski, 1896), emend. Liszka y Liszkowa, 1981

Lámina 1, Figura 24

Material: 1 espécimen del núcleo estratigráfico SS-10 (489,89 m).

Descripción: Conchilla unilocular, de contorno circular generalmente comprimida, con una periferia redondeada y centro deprimido. Pared de granos de grosor variable, compuesta de granos de cuarzo fino a medio ordenados. Aperturas entre los espacios de los granos o poros diminutos rebordeados por unos pocos granos a manera de labios.

Edad: Cretácico tardío (Cenomaniano) a Eoceno de acuerdo con Kaminski y Gradstein (2005).

\section{Discusión}

A diferencia de lo reportado en estudios regionales como los de Guzmán et al. (2004) o Rubio et al. (2009), este estudio muestra que hay una mayor riqueza de taxones aglutinantes de la reportada para esta unidad. Este aspecto se hace latente en SalazarOrtiz et al. (2020b), aunque dicho trabajo no presenta una discusión taxonómica detallada de las formas halladas. Igualmente, cabe resaltar que en todas las secciones estudiadas de nuestro trabajo, no se halló la especie Rzehakina epigona, un taxón ampliamente documentado en las sedimentitas de la Formación San Cayetano (Duque-Caro, 1967; 1968; Duque-Caro et al., 1996; Guzmán et al., 2004; Molinares y Jaramillo, 2005; Salazar-Ortiz et al., 2020b). Con el acervo de datos colectado en nuestro trabajo, desconocemos el motivo de la ausencia de $R$. epigona en el material estudiado, si bien posibles explicaciones pueden ser algún tipo de control ecológico (e.g. disponibilidad de nutrientes) o tafonómico. Por ende, estos aspectos refuerzan la necesidad de tener una idea más clara de qué características definen las asociaciones de foraminíferos aglutinados del Paleógeno en el CPSJ. A continuación, se hacen una serie de observaciones respecto al rango bioestratigráfico de la asociación encontrada en nuestro estudio, y las implicaciones que tiene esta en la interpretación paleoambiental de la Formación San Cayetano. 


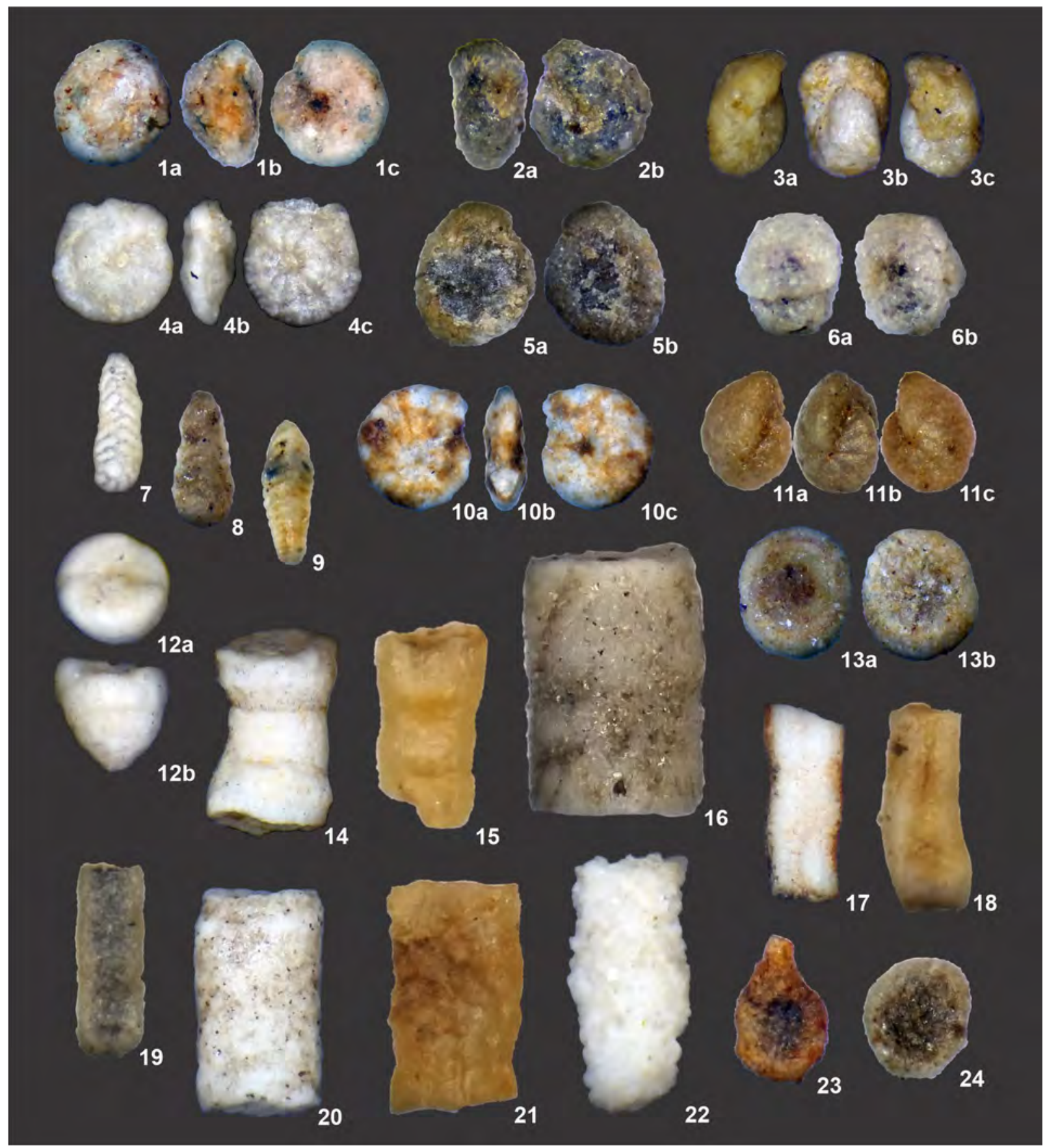

Lámina 1. Microfotografías de los foraminíferos bentónicos aglutinantes reportados en las localidades y núcleos estratigráficos del Paleógeno del CPSJ. 1a-c Ammoanita ruthvenmurrayi (Cushman y Renz) 2X. 2a-b Budashevaella multicamerata (Voloshinova y Budasheva) 3.5X. 3a-c Budashevaella trinitatensis (Cushman y Renz) 2X. 4a-c Haplophragmoides walteri (Grzybowski) 2.5X. 5a-b Trochamminoides proteus (Karrer emend. Rögl) 2X. 6a-b Trochamminoides variolarius (Grzybowski) 4X. 7-9 Spiroplectammina spectabilis (Grzybowski emend. Kaminski) 2X. 10a-c Reticulophragmium amplectens (Grzybowski) 3.5X. 11a-c Reticulophragmoides jarvisi (Thalmann, emend. Gradstein y Kaminski) 2.5X. 12a-b Marssonella oxycona aff. trinitatensis Cushman y Renz 4X. 13a-b Ammodiscus tenuissimus Grzybowski 1.8X. 14 Aschemocella subnodosiformis (Grzybowski em. Kaminski y Geroch) 1.2X. 15 Hormosina cf. velascoensis (Cushman, 1926) 1.2X. 16 Bathysiphon eocenicus Cushman y Hanna 0.8X. 17-18 Nothia excelsa (Grzybowski emend. Geroch y Kaminski) 1.2X. 19 Nothia latissima (Grzybowski) 2.5X. 20-21 Nothia robusta (Grzybowski) 0.8X. 22 Psammosiphonella discreta (Brady) 1x. 23 Saccammina grzybowskii (Schubert) 2.5X. 24 Psammosphaera irregularis (Grzybowski emend. Liszka y Liszkowa) 3.5X. 


\section{Edad de las asociaciones de foraminíferos aglutinados de la Formación San Cayetano}

La Figura 2 presenta los rangos estratigráficos de los taxones más representativos en las secciones estudiadas. Aunque muchas de las especies presentan rangos que vienen desde el Cretácico y otras son comunes a lo largo del Paleógeno, hay algunas que presentan rangos temporales cortos como Reticulophragmoides jarvisi (Thanetiano), o sus primeras/últimas ocurrencias ayudarían a restringir la edad de la asociación en su conjunto. Tal es el caso del límite YpresianoLutetiano (Eoceno temprano-medio) que podría ser definido a través de las extinciones de especies como Aschemocella subnodosiformis y Bathysiphon eocanicus, o por medio de las primeras ocurrencias estratigráficas de Budhashevaella multicamerata y Reticulophragmium amplectens (Figura 2). A su vez, hay especies como Nothia lattissima que estarían restringidas a sedimentitas no más jóvenes que el Paleoceno temprano (Daniano). Esto es significativo, considerando que el género Nothia parece ser un elemento constante cuando hay recobro únicamente de formas aglutinantes, y especies como $N$. robusta o $N$. excelsa presentan rangos bioestratigráficos bastante amplios. Si tenemos en cuenta los rangos bioestratigráficos de las especies que definen la zonación clásica de los trabajos de Duque-Caro (1967, 1968; Duque-Caro et al., 1996), observamos que tanto Spiroplectammina spectabilis (citada usualmente como S. grzybowskii) y Rzehakina epigona presentan rangos bioestratigráficos relativamente amplios (Cretácico tardío-Eoceno; Kaminski y Gradstein, 2005). En el caso de $R$. epigona, su última ocurrencia estratigráfica indica el Eoceno medio (Figura 2).

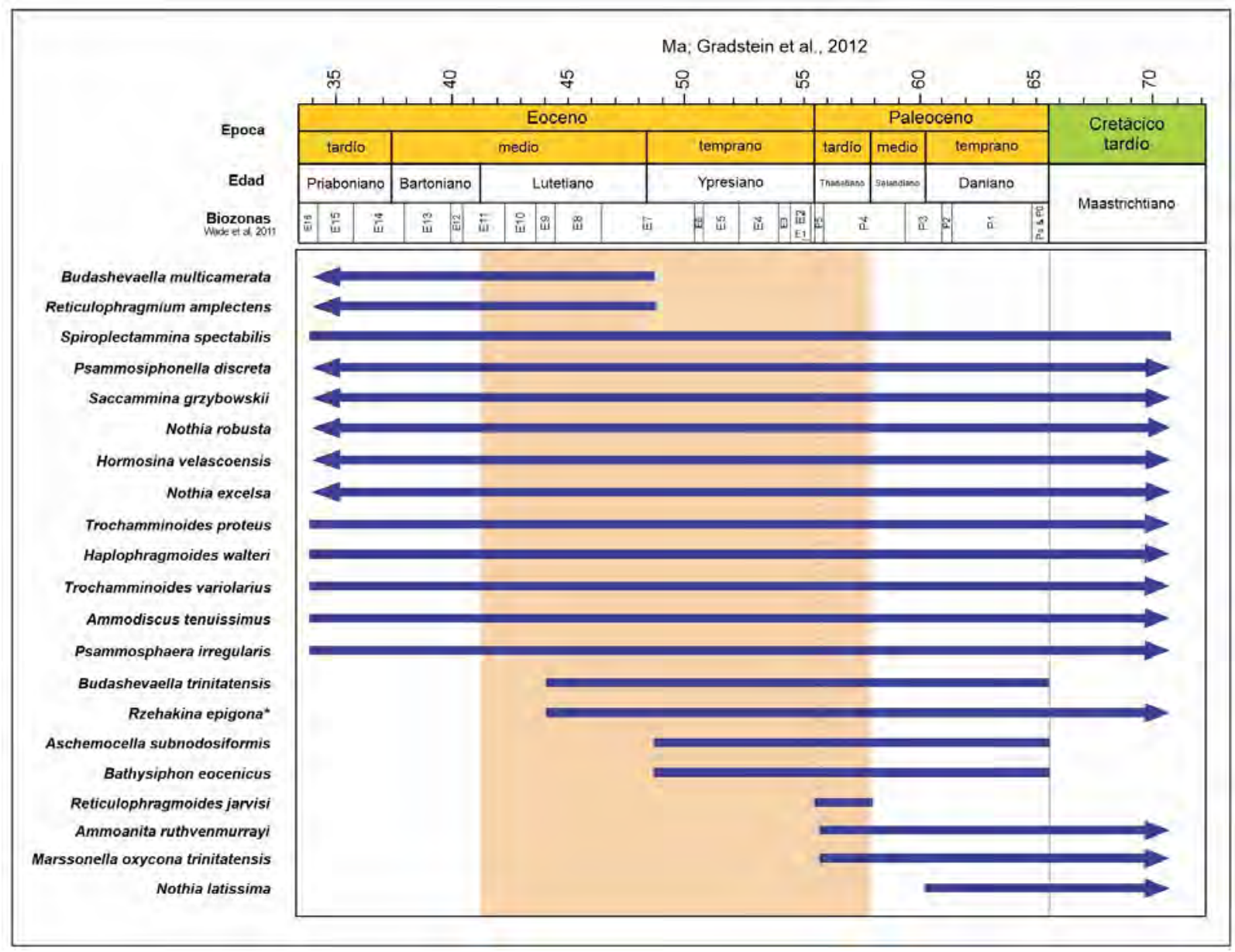

Figura 2. Rangos estratigráficos de foraminíferos bentónicos aglutinados reportados en localidades estudiadas del Paleógeno del CPSJ de acuerdo con Bolli et al. (1994) y Kaminski y Gradstein (2005). Se resalta el rango de edad de la Formación San Cayetano. *El rango bioestratigráfico de Rzehakina epigona (Rzehak) es incluido, si bien no se encontraron especímenes en las localidades estudiadas. 
Cabe resaltar que tanto los distintos estudios de Duque-Caro como trabajos regionales recientes (Guzmán et al., 2004; Molinares y Jaramillo, 2005; Rubio et al., 2009; SalazarOrtiz et al., 2020b), han buscado calibrar la edad de estos dos elementos diagnósticos a través de la identificación de formas planctónicas diagnósticas del Paleoceno tardío y el Eoceno temprano-medio (Morozavella aequa, Acarinina pentacamerata, Pseudoglobigerinella bolivariana; Olsson et al., 2006; Wade et al., 2011). Sin embargo, la presencia de formas plantónicas diagnósticas suele ser nula en muchas localidades de la Formación San Cayetano y eso dificulta una asignación de edad apropiada. Dificultades similares se han observado cuando se ha buscado integrar la información bioestratigrafía de los foraminíferos aglutinados con la de otros microfósiles (palinomorfos, nanofósiles, radiolarios; GEOTEC, 2003; Guzmán et al., 2004; Rubio et al., 2009; Salazar-Ortiz et al., 2020b).

Por ende, uno de los objetivos de este trabajo era presentar taxones adicionales que ayuden a definir la edad de las sedimentitas del Paleógeno del CPSJ, al no disponer de dichas formas diagnósticas. Por ejemplo, en ausencia de $R$. epigona, la extinción de Budhashevaella trinitatensis puede ser usada para definir el Eoceno medio, mientras que las últimas ocurrencias estratigráficas de Ammoanita ruthvenmurrayi y Marssonella oxycona trinitatensis ayudarían a definir el límite Paleoceno-Eoceno (Figura 2).

Teniendo en cuenta la información consignada en este estudio, los rangos estratigráficos de las especies identificadas son consecuentes con lo reportado en la literatura para la Formación San Cayetano. Dichos trabajos proponen una edad no más antigua que el Paleoceno tardío y no más joven que Eoceno medio. Tal es el caso de la publicación más reciente de Salazar et al. (2020b), la cual estudió distintas secciones de la Formación San Cayetano en el sector de Luruaco y basados en la información bioestratigráfica integrada de foraminíferos (planctónicos y bentónicos), nanofósiles y palinomorfos sugieren una edad no más joven que el Bartoniano (Eoceno medio) para los segmentos más superiores de la Formación San Cayetano.

Teniendo en cuenta que Salazar-Ortiz et al. (2020b) claramente establece cuatro segmentos para la unidad, es importante que en un futuro se explore a detalle la posición estratigráfica y distribución espacial de cada uno de los eventos de foraminíferos que se han reportado hasta el momento. De esta manera se podrá evaluar la importancia bioestratigráfica tanto de taxones de referencia como $R$. epigona y $S$. spectabilis, como de las especies aglutinadas que se compilaron en este estudio.

\section{Significado paleoambiental de las asociaciones de foraminíferos aglutinados de la Formación San Cayetano}

Teniendo en cuenta los morfogrupos encontrados en las secciones estudiadas, se exploró su significado paleoambiental a través de la definición de morfotipos, utilizando los parámetros planteados tanto para asociaciones de foraminíferos aglutinados recientes, como del Paleógeno (Figura 3; Cetean et al., 2011; Murray et al., 2011; Benedetti, 2017; Setoyama et al., 2017). Hay que tener en cuenta que en el rango de edad en el que se depositó la Formación San Cayetano, hubo un evento de perturbación climática (PaleoceneEocene Thermal Maximum, PETM), el cual está ampliamente documentado en los fondos oceánicos y que para los foraminíferos bentónicos dicho evento constituyó un evento de extinción bastante significativo (30-50\% del total de los grupos; Alegret et al., 2018, 2021; Hayek et al., 2019). Por ende, las estimaciones paleoambientales basadas en morfotipos de foraminíferos bentónicos del Paleógeno, presentan un margen de incertidumbre mayor que a las planteadas para asociaciones posteriores a la transición climática que se registra globalmente en el límite EocenoOligoceno (Hutchinson et al., 2021).

En general, el predominio y abundancia de formas aglutinantes tubulares como Bathysiphon o Nothia, que corresponden a organismos suspensívoros, está asociado a entornos propios de zonas distales y abanicos submarinos (flysch-type assemblages; Kaminski y Gradstein, 2005; Murray et al., 2011). Por ende, las asociaciones encontradas en nuestro estudio fueron comparadas con las reportadas en depósitos turbidíticos del Paleógeno en Trinidad (Kaminski et al., 1988). Aun cuando la representación de organismos tubulares es alta ( 25\% de la asociación total), estos autores encuentran una mayor variedad de otros morfotipos, respecto a lo que reportamos para las sedimentitas de la Formación San Cayetano. En nuestro caso, el predominio del morfotipo tubular podría ser explicado por una mayor inestabilidad del sustrato, siendo mayor el aporte de corrientes turbidíticas, variaciones en la paleoproductividad o condiciones marinas profundas más proximales al continente. Sin embargo, no se descarta algún tipo de control tafonómico. Aunque su presencia sea menor, la presencia de otros morfotipos (espiral aplanado, globular), pero con otras estrategias de adaptación (detritívoros activos y pasivos) reforzarían la idea de una mayor complejidad en las condiciones ambientales de las localidades estudiadas (Figura 3). 


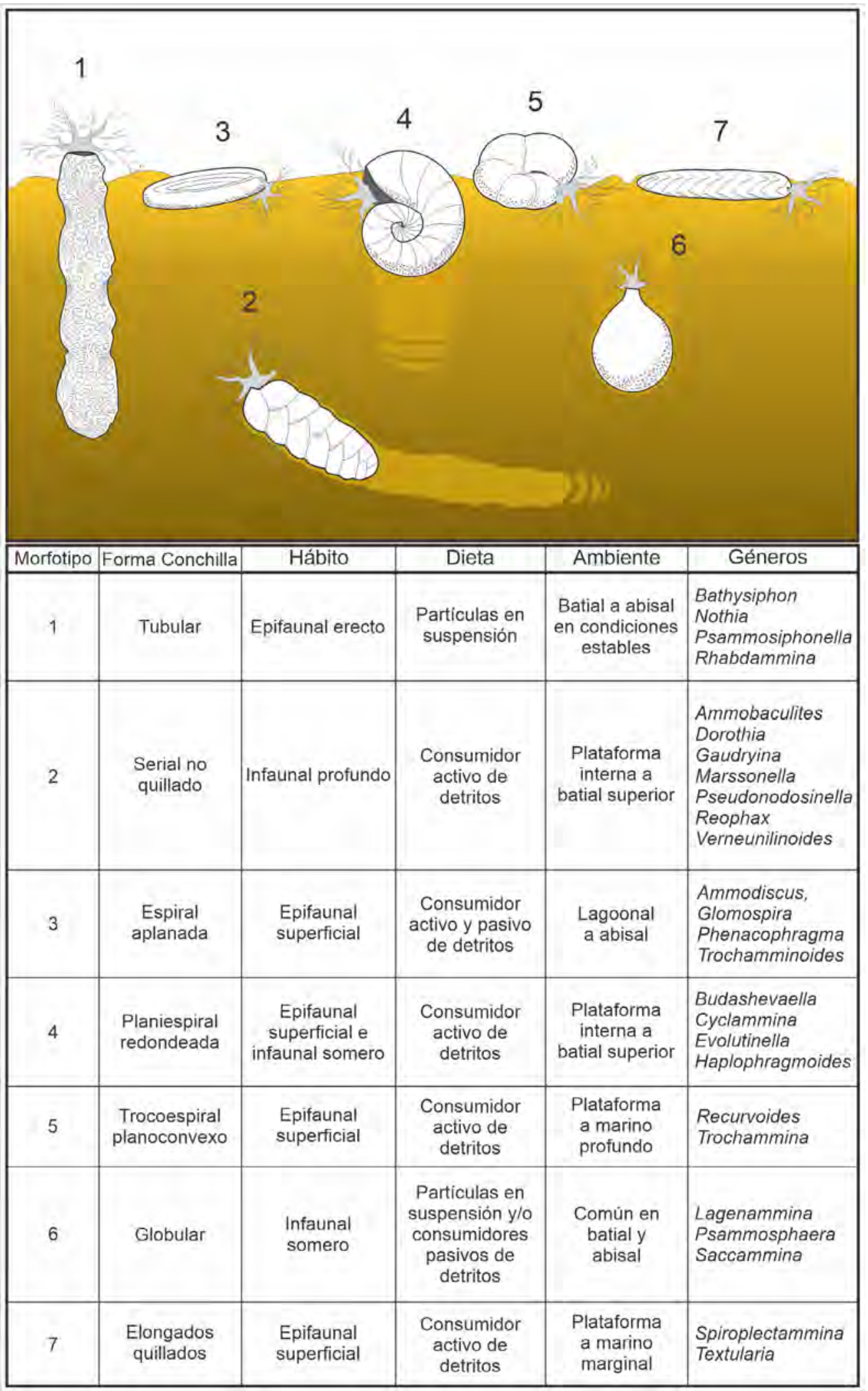

Figura 3. Morfotipos de foraminíferos bentónicos aglutinados reportados en localidades y pozos del Paleógeno del CPSJ durante el Paleógeno. Morfotipos basados en Benedetti (2017), Cetean et al. (2011), Murray et al. (2011) y Setoyama et al. (2017). 
Ahora, desde un punto de vista general, esta interpretación paleoambiental de entornos turbidíticos coincide con las propuestas en sucesiones turbidíticas de Trinidad o los Cárpatos (Kaminski et al., 1988; Waśkowska, 2021), y las ya referidas en los distintos trabajos de Duque-Caro (1967, 1968; Duque-Caro et al., 1996) y de otros autores en secciones de la Formación San Cayetano. Sin embargo, desde los trabajos de GEOTEC (2003), Guzmán et al. (2004) y publicaciones posteriores (e.g. Bermúdez et al., 2009), la interpretación clásica de un ambiente deposicional exclusivamente turbidítico viene siendo revisada, e incluso descartada en distintos segmentos de la Formación San Cayetano. Como se ha mencionado previamente, el trabajo de Salazar-Ortiz et al. (2020b) corresponde a la revisión más reciente del contexto litoestratigráfico del Paleógeno en el CPSJ. Dichos autores proponen que las sedimentitas de la Formación San Cayetano se depositaron en un sistema deltaico retrogradante, con subambientes asociados de prodelta y flujos hiperpícnicos. Elementos como la presencia de glauconita, al igual que restos vegetales (macrofósiles y palinomorfos) e icnofósiles refuerzan la idea de una depositación somera y un aporte continental proximal. Sin embargo, los mismos autores advierten que dichas facies pueden encontrarse en abanicos submarinos a profundidades mayores y que por ende las estimaciones de la paleobatimetría aún no son concluyentes.

Por tal motivo, se recalca en la necesidad de que los futuros trabajos en la Formación San Cayetano sigan integrando la información sedimentológica obtenida en campo, con las interpretaciones paleoambientales que sugieran los distintos niveles fosilíferos (macrofósiles y microfósiles) encontrados. Los estudios micropaleontológicos a futuro deben favorecer la recolección de datos cuantitativos (abundancias, distribución espacial) y un amarre estratigráfico claro. Solo de esa forma se podrá no solo calibrar las interpretaciones basadas en las asociaciones de foraminíferos aglutinados, sino también entender las causas de su distribución espacial y estratigráfica en el CPSJ (e.g. pulsos de paleoproductividad, sistemas turbidíticos).

\section{Conclusiones}

Pese a la poca variabilidad de las asociaciones de foraminíferos aglutinados reportadas previamente para la Formación San Cayetano, este estudio resalta que hay una mayor complejidad en las morfoespecies encontradas en esta unidad. Por ende, se elaboró una descripción taxonómica detallada de las especies más representativas. En general, las asociaciones de foraminíferos encontradas coinciden en asignar un rango de edad de Paleoceno tardío-Eoceno medio para las sedimentitas de la Formación San Cayetano. Sin embargo, taxones comoAschemocella subnodosiformis, Bathysiphon eocanicus, Budhashevaella trinitatensis, Nothia lattissima, Reticulophragmoides jarvisi, entre otros, podrían ayudar a refinar la edad de los niveles fosilíferos donde se encuentren. A su vez, el tipo de morfotipos encontrados, coincide con interpretaciones previas que les atribuyen un ambiente distal, con una inestabilidad de fondo posiblemente asociada a flujos turbidíticos. Teniendo en cuenta que diversos estudios paleontológicos y sedimentológicos cuestionan esta interpretación clásica para algunos de los segmentos descritos en la Formación San Cayetano, se hace necesaria la apropiada integración de estos trabajos con aquellos que a futuro describan niveles con foraminíferos. Esto, para tener no solo una idea completa del sentido estratigráfico y paleoambiental de dichos niveles, sino también generar una discusión acerca de la distribución espacial de las sedimentitas del Paleógeno en el CPSJ.

\section{Agradecimientos}

Los autores agradecen a HOCOL S.A. por la autorización para presentar los resultados de los análisis bioestratigráficos que se vienen adelantando en el Cinturón Plegado de San Jacinto desde 2017.

\section{Referencias}

Alegret, L.; Reolid, M.; Vega-Pérez, M. (2018). Environmental instability during the latest Paleocene at Zumaia (Basque-Cantabric Basin): the bellwether of the Paleocene-Eocene Thermal Maximum. Palaeogeography, Palaeoclimatology, Palaeoecology, 497, 186-200. https://doi. org/10.1016/j.palaeo.2018.02.018

Alegret, L.; Arreguín-Rodríguez, G.J.; TrasviñaMoreno, C.A.; Thomas, E. (2021). Turnover and stability in the deep sea: Benthic foraminifera as tracers of Paleogene global change. Global and Planetary Change, 196. https://doi.org/10.1016/j. gloplacha.2020.103372

Benedetti, A. (2017). Eocene/Oligocene deep-water agglutinated foraminifers (DWAF) assemblages from the Madonie Mountains (Sicily, Southern 
Italy). Palaeontologia Electronica, 20.1, 1-66. https://doi.org/10.26879/660

Bermúdez, H.D.; Grajales, J.A.; Restrepo, L.C.; Rosero, J.S. (2009). Estudio Integrado de los núcleos y registros obtenidos de los pozos someros tipo "slim holes" en la Cuenca del Sinú. Tomo 1, Anexo 1. Bogotá. Agencia Nacional de Hidrocarburos - Universidad de Caldas.

Bolli, H.M.; Beckmann, J.P.; Saunders, J.B. (1994). Benthic Foraminiferal biostratigraphy of the South Caribbean region. Cambridge University Press. https://doi.org/10.1017/S0016756800021324

Bordine, B.W. (1974). Neogene bioestratigraphy and paleoenvironments, Lower Magdalena Basin, Colombia. Ph.D. Thesis, Louisiana State University and Agricultural and Mechanical College, Ann Arbor, USA.

Brady, H.B. (1881). Notes on some of the reticularian Rhizopoda of the "CHALLENGER" Expedition; Part III. Quarterly Journal of Microscopical Sciences, 21, 31-71.

Catuneanu, O.; Abreu, V.; Bhattacharya, J.P.; Blum, M.D.; Dalrymple, R.W.; Eriksson, P.G.; Fielding, C.R.; Fisher, W.L.; Galloway, W.E.; Gibling, M.R.; Giles, K.A.; Holbrook, J.M.; Jordan, R.; Kendall, C.G.St.C.; Macurda, B.; Martinsen, O.J.; Miall, A.D.; Neal, J.E.; Nummedal, D.; Pomar, L.; Posamentier, H.W.; Pratt, B.R.; Sarg, J.F.; Shanley, K.W.; Steel, R.J.; Strasser, A.; Tucker, M.E.; Winker, C. (2009). Towards the standardization of sequence stratigraphy. EarthScience Reviews, 92(1-2), 1-33. https://doi. org/10.1016/j.earscirev.2008.10.003

Cetean, C.G.; Balc, R.; Kaminski, M.A.; Filipescu, S. (2011). Integrated biostratigraphy and palaeoenvironments of an upper Santonian upper Campanian succession from the southern part of the Eastern Carpathians, Romania. Cretaceous Research, 32(5), 575-590. https://doi. org/10.1016/j.cretres.2010.11.001

Chenevart, C.H. (1963). Les dorsales transverses anciennes de Colombie et leurs homologues d'Amerique Latine. Eclogae Geologic Helvetiae, 52(2), 907-927.
Cushman, J.A. (1926). The foraminifera of the Velasco Shale of the Tampico Embayment. AAPG Bulletin, 10(6), 581-612. https:// doi.org/10.1306/3D932728-16B1-11D78645000102C1865D

Cushman, J.A.; Hanna, G.D. (1927). Foraminifera from the Eocene near Coalinga, California. Proceedings of the California Academy of Sciences, 4, 205-229.

Cushman, J.A.; Renz, H.H. (1946). The foraminiferal fauna of the Lizard Springs Formation of Trinidad, British West Indies. Cushman Laboratory for Foraminiferal Research.

de Porta, J. (1970). On planktonic foraminiferal zonation in the tertiary of Colombia. Micropaleontology, 16(2), 216-220. https://doi.org/10.2307/1485115

Duque-Caro, H. (1967). Informe bioestratigráfico preliminar de los cuadrángulos E-8 y D-8, Informe 1522. Servicio Geológico Nacional. Bogotá.

Duque-Caro, H. (1968). Observaciones generales a la bioestratigrafía y geología regional en los departamentos de Bolívar y Córdoba. Boletín de Geología, 24, 71-87.

Duque-Caro, H. (1971a). Ciclos tectónicos y sedimentarios en el norte de Colombia y sus relaciones con la paleoecología. Boletín Geológico, 19(3), 1-23.

Duque-Caro, H. (1971b). Relaciones entre la bioestratigrafía y la cronoestratigrafía en el llamado Geosinclinal de Bolívar. Boletín Geológico, 19(3), 25-68.

Duque-Caro, H. (1973). Foraminíferos planctónicos y el terciario de Colombia. Informe 1651. INGEOMINAS. Bogotá.

Duque-Caro, H. (1975). Los foraminíferos planctónicos y el Terciario de Colombia. Revista Española de Micropaleontología, 7(3), 403-427.

Duque-Caro, H. (1979). Major structural elements and evolution of northwestern Colombia. In: J.S. Watkins, L. Montadert, P. Wood-Dickerson (eds.). Geological and geophysical investigations of 
continental margins (pp. 329-351). Vol. 29. AAPG Memoir. https://doi.org/10.1306/M29405C22

Duque-Caro, H. (1991). Contributions to the geology of the Pacific and the Caribbean coastal areas of northwestern Colombia and South America. Ph.D. Thesis, Princeton University, USA.

Duque-Caro, H.; Guzmán, G.; Hernández, O. (1996). Geología de la plancha 38. Informe No. 2188. INGEOMINAS. Bogotá.

GEOTEC. (2003). Geología de los Cinturones Sinú - San Jacinto, planchas 50 Puerto Escondido, 51 Lorica, 59 Mulatos, 60 Canalete, 61 Montería, 69 Necoclí, 70 San Pedro de Urabá, 71 Planeta Rica, 79 Turbo, 80 Tierralta. Escala 1:100.000 Memoria Explicativa. INGEOMINAS. Bogotá.

Geroch, S.; Kaminski, M.A. (1992). The morphology and systematics of Nothia excelsa (Grzybowski), a deep-water agglutinated foraminifer. Rocznik Polskiego Towarzystwa Geologicznego, 62, 255265.

Gómez, J.; Montes, M.E. (2020). Mapa geológico de Colombia 2020. Escala 1:1.000.000. Servicio Geológico Colombiano.

Gradstein, F.M.; Kaminski, M.A. (1989). Taxonomy and biostratigraphy of new and emended species of Cenozoic deep-water agglutinated foraminifera from the Labrador and North Seas. Micropaleontology, 35, 72-92. https://doi. org/10.2307/1485538

Grzybowski, J. (1896). Otwornice czerwonych ilów z Wadowic. Rozprawy Wydziału MatematycznoPrzyrodniczego, Akademia Umiejętności $w$ Krakowie, 2(30), p. 273, pl. 8, figs. 12-13.

Grzybowski, J. (1898). Otwornice pokładow naftonośnych okolicy Krosna. Rozprawy Wydziału Matematyczno-Przyrodniczego, Akademia Umiejętności w Krakowie, 33, p. 290, pl. 11, fig. 31.

Guzmán, G.; Gómez, E.; Serrano, B. (2004). Geología de los cinturones del Sinú, San Jacinto y borde occidental del Valle Inferior del Magdalena, Caribe colombiano. Escala 1:300.000. INGEOMINAS. Bogotá.
Hayek, L.A.; Buzas, M.; Thomas, E. (2019). Identifying disruptions to the ecological balance of nature: a foraminiferal example across the initiation of the Paleocene-Eocene Thermal Maximum. Paleobiology, 45(1), 98-113. https:// doi.org/10.1017/pab.2018.45

Hutchinson, D.K.; Coxall, H.K.; Lunt, D.J.; Steinthorsdottir, M.; de Boer, A.M.; Baatsen, M.; von der Heydt, A.; Huber, M.; Kennedy-Asser, A.T.; Kunzmann, L.; Ladant, J-B.; Lear, C.H.; Moraweck, K.; Pearson, P.N.; Piga, E.; Pound, M.J.; Salzmann, U; Scher, H.D.; Sijp, W. P.; Sliwinska, K.K.; Wilson, P.A.; Zhang, Z. (2021). The Eocene-Oligocene transition: a review of marine and terrestrial proxy data, models and model-data comparisons. Climate of the Past, 17(1), 269-315. https://doi.org/10.5194/cp-17269-2021

Kaminski, M.A. (1984). Shape variation in Spiroplectammina spectabilis (Grzybowski). Acta Paleontologica Polonica, 29, 29-49.

Kaminski, M.A.; Geroch, S. (1993). A revision of foraminiferal species in the Grzybowski Collection. Grzybowski Foundation Special Publication, 1, 239-323.

Kaminski, M.A.; Gradstein, F.M. (2005). Atlas of Paleogene cosmopolitan deep-water agglutinated Foraminifera. Grzybowski Foundation.

Kaminski, M.A.; Gradstein, F.M.; Berggren, W.A.; Geroch, S.; Beckmann, J.P. (1988). Flyschtype agglutinated foraminiferal assemblages from Trinidad: Taxonomy, stratigraphy and paleobathymetry. Abhandlungen Geologie Bundesanstalt, 41, 155-227.

Karrer, F. (1866). Über das Auftreten von Foraminiferen in den älteren Schichten des Wiener Sandsteins. Sitzungsber. k. Akad. Wiss. math.-naturwiss. Kl. 52, 492-497. p. 494, pl. 1, fig 8.

Liszka, S.; Liszkowa, J. (1981). Revision of $J$. Grzybowski's paper (1896) "Foraminifera of the red clays from Wadowice”. Rocznik Polskiego Towarzystwa Geologicznego.

Loeblich, A; Tappan, H. (1987). Foraminiferal genera and their classification. Van Nostrand Reinhold Company. 
Molinares, C.; Jaramillo, C. (2005). Biostratigraphic value of Rzehakina epigona in the PaleoceneEarly Eocene of Northern South America. 7th International Workshop about Agglutinated Foraminifera. Urbino, Italy.

Molinares, C.E.; Martinez, J.I.; Fiorini, F.; Escobar, J; Jaramillo, C. (2012). Paleoenvironmental reconstruction for the lower Pliocene Arroyo Piedras section (Tubará - Colombia): Implications for the Magdalena River - paleodelta's dynamic. Journal of South American Earth Sciences, 39, 170-183. https://doi.org/10.1016/j. jsames.2012.04.007

Mora, J.A.; Ibáñez-Mejia, M.; Oncken, O.; De Freitas, M.; Vélez, V.; Mesa, A.; Serna, L. (2017a). Structure and age of the Lower Magdalena Valley Basin basement, northern Colombia: New reflection-seismic and U-Pb-Hf insights into the termination of the central Andes against the Caribbean Basin. Journal of South American Earth Sciences, 74, 1-26. https://doi. org/10.1016/j.jsames.2017.01.001

Mora, J.A.; Oncken, O.; Le Breton, E.; IbáñezMejia, M.; Faccenna, C.; Veloza, G.; Vélez, V.; De Freitas, M.; Mesa, A. (2017b). Linking Late Cretaceous to Eocene tectonostratigraphy of the San Jacinto fold belt of NW Colombia with Caribbean plateau collision and flat subduction. Tectonics, 36(11), 2599-2629. https://doi. org/10.1002/2017TC004612

Murray, J.W.; Alve, E.; Jones, B.W. (2011). A new look at modern agglutinated benthic foraminiferal morphogroups: their value in palaeoecological interpretation. Palaeogeography, Palaeoclimatology, Palaeoecology, 309(34), 229-241. https://doi.org/10.1016/j. palaeo.2011.06.006

Olsson, R.K.; Berggren, W.A.; Hemleben, C.; Huber, B.T. (2006). Atlas of Eocene planktonic foraminifera. Smithsonian Institution Press. https://doi.org/10.5479/si.00810266.85.1

Osorio-Granada, E.; Pardo-Trujillo, A.; RestrepoMoreno, S.A.; Gallego, F.; Muñoz, J.; Plata, A.; Trejos-Tamayo, R.; Vallejo, F.; BarbosaEspitia, A.; Cardona-Sánchez, F.J.; Foster, D.A.; Kamenov, G. (2020). Provenance of EoceneOligocene sediments in the San Jacinto Fold Belt:
Paleogeographic and geodynamic implications for the northern Andes and the southern Caribbean. Geosphere, 16(1), 210-228. https:// doi.org/10.1130/GES02059.1

Patarroyo, G.; Torres, G.; Veloza, G.; Mora, A.; Gómez, D.F. (2019). Asociaciones de foraminíferos del Paleógeno inferior en el Cinturón Plegado de San Jacinto. XVII Congreso Colombiano de Geología. Santa Marta, Colombia.

Petters, V.; Sarmiento, S. (1956). Oligocene and Lower Miocene bioestratigraphy of the CarmenZambrano area, Colombia. Micropaleontology, 2(1), 7-35. https://doi.org/10.2307/1484490

Redmond, C.D. (1953). Miocene Foraminifera from the Tubara beds of Northern Colombia. Journal of Paleontology, 27(5), 708-733.

Rincón, D.A.; Arenas, J.E.; Cuartas, C.H.; Cárdenas, A.L.; Molinares, C.E.; Caicedo, C.; Jaramillo, C. (2007). Eocene-Pliocene planktonic foraminifera biostratigraphy from the continental margin of the southwest Caribbean. Stratigraphy 4(4), 261-311.

Rögl, F. (1995). A Late Cretaceous flysch-type agglutinated foraminiferal fauna from the Trochamminoides proteus type locality (Wien - Hütteldorf, Austria). Grzybowski Foundation Special Publication, 3, 249-263. p. 255, pl. 2, figs. $1-6$.

Rubio, M.A.; Herrera, J.M.; Navarrete, R.E.; Bermúdez, H.D.; Calderón, J.E.; Parra, F.J.; Sarmiento, G.; Vega, F.; Perrilliat, M.C. (2009). Cartografía geológica, levantamiento de columnas estratigráficas, toma de muestras y análisis bioestratigráficos. Sector de Chalán (Cuenca Sinú-San Jacinto). Informe Geológico para la Agencia Nacional de Hidrocarburos. ATG Asesorías Técnicas Geológicas. 93 p.

Salazar-Ortiz, E.A.; Rincón-Martínez, D.; Páez, L.A.; Restrepo, S.M.; Barragán, S. (2020a). Middle Eocene mixed carbonate-siliciclastic systems in the southern Caribbean (NW Colombian margin). Journal of South American Earth Sciences, 99. https://doi.org/10.1016/j.jsames.2020.102507

Salazar-Ortiz, E.A.; Numpaque, J.; Bernal, L.; Ocampo, E.; Matajira, A.; Villabona, J.; Gómez, D.; García, G.; Méndez, S.; Martínez, E.; Sánchez, 
D.; Sotelo, A.P.; Aguirre, L. (2020b). Geología del área Sinú - San Jacinto, Planchas 23, 24, 30, 31 y parte de las planchas 37 y 38 a escala 1:50.000. Servicio Geológico Colombiano. Bogotá. https:// doi.org/10.13140/RG.2.2.22668.23683

Schubert, R.J. (1902). Neue und interessante Foraminiferen aus dem südtiroler Alttertiär. Beiträge zur Paläontologie und Geologie Österreich-Ungarns und des Orients, 14, 9-26.

Setoyama, E.; Kaminski, M.A.; Jarosław, T. (2017). Late Cretaceous-Paleogene foraminiferal morphogroups as palaeoenvironmental tracers of the rifted Labrador margin, northern protoAtlantic. Grzybowski Foundation Special Publication, 22, 179-220.

Stone, B. (1968). Planktonic foraminiferal zonation in the Carmen-Zambrano area, Colombia. Micropaleontology, 14(3), 363-364.

Thalmann, H.E. (1932). Nonion jarvisi nom. nov. and Trochammina kellettae nom. nov. Ecologae Geologicae Helvetiae, 25, 312-313.

Vail, P.R.; Todd, R.G.; Sangree, J.B. (1977). Seismic stratigraphy and global changes of sea level,
Part 5: Chronostratigraphic significance of seismic reflections. In: C.E. Payton (ed.). Seismic stratigraphy - applications to hydrocarbon exploration (pp. 99-116). Vol. 26. AAPG Memoir. https://doi.org/10.1306/M26490C7

Voloshinova, N.A.; Budasheva, A.I (1961). Lituolids and Trochamminids from the Tertiary deposits of Sakhalin Island and the Kamchatka Peninsula (In Russian). Trudy VNIGRI, 170 (12), p. 201, pl. 7, figs $6 \mathrm{a}-\mathrm{c}$, pl. 8, figs 1a-c, 6a-c.

Wade, B.S.; Pearson, P.N.; Berggren, W.A.; Pälike, H. (2011). Review and revision of Cenozoic tropical planktonic biostratigraphy and calibration to the geomagnetic polarity and astronomical time scale. Earth Science Reviews, 104(104), 111-142. https://doi.org/10.1016/j.earscirev.2010.09.003

Waśkowska, A. (2021). Agglutinated Foraminiferal Acmes and Their Role in the Biostratigraphy of the Campanian-Eocene Outer Carpathians. Geosciences, 11(9). https://doi.org/10.3390/ geosciences 11090367

Fecha de recibido: 21 de julio de 2021 Fecha de aprobado: 18 de noviembre de 2021 